\title{
On compact spaces carrying Radon measures of uncountable Maharam type
}

\author{
by
}

\author{
D. H. Frem lin (Colchester)
}

\begin{abstract}
If Martin's Axiom is true and the continuum hypothesis is false, and $X$ is a compact Radon measure space with a non-separable $L^{1}$ space, then there is a continuous surjection from $X$ onto $[0,1]^{\omega_{1}}$.
\end{abstract}

1. Introduction. For any probability space $(X, \Sigma, \mu)$, its measure algebra is the quotient Boolean algebra $\Sigma / \mathcal{N}$, where $\mathcal{N}$ is the $\sigma$-ideal of sets of measure 0 . For more than fifty years we have had a complete description of these Boolean algebras. There is the two-element algebra $\{0,1\}$; for each infinite cardinal $\kappa$ there is the measure algebra $\mathfrak{B}_{\kappa}$ of the usual measure on $\{0,1\}^{\kappa}$; and there are countable products of these of the form $\mathcal{P} J \times \prod_{i \in I} \mathfrak{B}_{\kappa_{i}}$, where $I$ and $J$ are countable sets and $\left\langle\kappa_{i}\right\rangle_{i \in I}$ is a family of distinct infinite cardinals. And that is all. (See [12] and [5], §3.) Part of the interest of this classification lies in the fact that it completely describes the function spaces $L^{p}(\mu)$, for $1 \leq p \leq \infty$, up to Banach lattice isomorphism.

Now suppose that $(X, \mathfrak{T})$ is a topological space. In this case a measure on $X$ may or may not be related to the topology in various ways. By far the most important is the idea of Radon measure, in which all open sets (and therefore all Borel sets) are measurable and $\mu E=\sup \{\mu K: K \subseteq E$ is compact\} for every measurable set $E$. It is customary, in this context, to suppose that $X$ is Hausdorff, so that compact sets are closed. There are complications if $\mu X=\infty$; in this paper I will consider probability measures exclusively. Now, given a Hausdorff space $(X, \mathfrak{T})$, we can ask: which Boolean algebras can appear as the measure algebras of Radon probability measures on $X$ ? Write $\mathrm{K}(X)$ for the set of infinite cardinals $\kappa$ for which there is a Radon probability measure $\mu$ on $X$ such that the measure algebra of $\mu$ is isomorphic to $\mathfrak{B}_{\kappa}$. It is known that if $\omega \leq \lambda \leq \kappa \in \mathrm{K}(X)$ then $\lambda \in \mathrm{K}(X)$,

1991 Mathematics Subject Classification: 28C15, 54A25. 
so that $\mathrm{K}(X)$ must be an initial segment of the class of infinite cardinals. It may or may not contain its supremum, but once we know $\mathrm{K}(X)$ we can determine all the possible measure algebras of Radon probability measures on $X$, as follows. If $X=\emptyset$ there are none. If $X$ is finite and not empty, we get algebras of the form $\mathcal{P} J$ where $1 \leq \#(J) \leq \#(X)$. If $X$ is infinite, we get algebras of the form $\mathcal{P} J \times \prod_{i \in I} \mathfrak{B}_{\kappa_{i}}$, where $I$ and $J$ are countable sets and $\left\langle\kappa_{i}\right\rangle_{i \in I}$ is a family of distinct members of $\mathrm{K}(X)$; and these lists are complete.

So we turn to the determination of $\mathrm{K}(X)$. Because Radon measures are defined by their behaviour on compact sets, $\mathrm{K}(X)=\bigcup\{\mathrm{K}(K): K \subseteq X$ is compact\}, so we begin by investigating compact spaces $X$. Now if $X$ and $Y$ are compact Hausdorff spaces and $f: X \rightarrow Y$ is a continuous surjection, then $\mathrm{K}(Y) \subseteq \mathrm{K}(X)$ ([7], Prop. 2.1); if $Z$ is a closed subset of $X$, then $\mathrm{K}(Z) \subseteq \mathrm{K}(X)$; further, $\sup \mathrm{K}(X) \leq w(X)$, the topological weight of $X$; and $\kappa \in \mathrm{K}\left([0,1]^{\kappa}\right)$ for any infinite cardinal $\kappa$. What this means is that $\mathrm{K}\left([0,1]^{\kappa}\right)$ must be just $\{\lambda: \omega \leq \lambda \leq \kappa\}$, and if there is a continuous function from $X$ onto $[0,1]^{\kappa}$ then $\kappa \in \mathrm{K}(X)$.

The question now arises: is there a converse to this result? If we know that $\kappa \in \mathrm{K}(X)$, when, if ever, can we deduce that there is a continuous surjection from $X$ onto $[0,1]^{\kappa}$ ? I will say that an infinite cardinal $\kappa$ has Haydon's property if whenever $X$ is a compact Hausdorff space and $\kappa \in \mathrm{K}(X)$, then there is a continuous surjection from $X$ onto $[0,1]^{\kappa}$. Of course, $\omega$ has Haydon's property, since for any compact Hausdorff space $X$ with a non-empty perfect subset there is a continuous surjection from $X$ onto $[0,1]^{\omega}$. The first investigation of the question was by R. G. Haydon, who showed that if $\kappa$ is regular and $\lambda^{\omega}<\kappa$ for every $\lambda<\kappa$ (for instance, if $\kappa=\mathfrak{c}^{+}$), then $\kappa$ has Haydon's property ([7], Theorem 2.4). Recently, G. Plebanek extended Haydon's result by showing that it is true for any cardinal $\kappa$ such that $\operatorname{cf}(\kappa) \geq \omega_{2}$ and $\kappa$ is a precaliber of $\mathfrak{B}_{\kappa}$ ([14], Theorem 4.1).

These results leave open the basic case $\kappa=\omega_{1}$. In [8], Haydon gave an example to show that if the continuum hypothesis is true then $\omega_{1}$ does not have Haydon's property. This example has been refined and adapted in various ways $([10],[2])$. In the present context, the best results are due to K. Kunen and J. van Mill, who showed that if $[0,1]^{\omega_{1}}$ can be covered by $\omega_{1}$ negligible sets then $\omega_{1}$ does not have Haydon's property $([11])$, and to Plebanek, who showed that if $\kappa$ is a cardinal of uncountable cofinality which is not a precaliber of $\mathfrak{B}_{\kappa}$ then $\kappa$ does not have Haydon's property ([14], Theorem 4.2). M. R. Burke has pointed out that if we add $\omega_{2}$ random reals to a model of ZFC+CH then $\omega_{1}$ becomes a precaliber of $\mathfrak{B}_{\omega_{1}}$, but $\omega_{1}$ does not have Haydon's property because the conditions of Theorem 6.2 of [14] are satisfied. 
All these examples have served to concentrate attention on Martin's Axiom: what happens if Martin's Axiom is true and the continuum hypothesis is false? In this paper I show that under these circumstances $\omega_{1}$ does have Haydon's property; and in fact the same is true of any infinite cardinal $\kappa$ such that $\mathrm{MA}(\kappa)$ is true (Theorem 9 below).

2. Notation. I follow [3] in writing $\mathfrak{m}$ for the least cardinal such that $\mathrm{MA}(\mathfrak{m})$ is false, so that Martin's Axiom becomes " $\mathfrak{m}=\mathfrak{c}$ ". If $I$ is a set, $\kappa$ a cardinal then $[I]^{\kappa}$ is the set of subsets of $I$ of cardinal $\kappa$, and $[I]^{<\omega}=$ $\bigcup_{n \in \mathbb{N}}[I]^{n}$ is the set of finite subsets of $I$. If $\mathfrak{A}$ is a Boolean algebra, a cardinal $\kappa$ is a precaliber of $\mathfrak{A}$ if for every family $\left\langle a_{\alpha}\right\rangle_{\alpha<\kappa}$ of non-zero elements of $\mathfrak{A}$, there is a set $A \in[\kappa]^{\kappa}$ such that $\inf _{\xi \in I} a_{\xi} \neq 0$ for every non-empty finite $I \subseteq A$. Note that if $\kappa<\mathfrak{m}$ has uncountable cofinality then $\kappa$ is a precaliber of every ccc Boolean algebra ([3], 41Ca), and that $\omega_{1}$ is a precaliber of $\mathfrak{B}_{\omega_{1}}$ iff $[0,1]^{\omega_{1}}$ is not the union of $\omega_{1}$ negligible sets (use [6], A2U).

In a Boolean algebra $\mathfrak{A}$, I will use the symbol $\cap$ to represent "intersection" (the "product" when $\mathfrak{A}$ is regarded as a ring); $1 \backslash a$ will be the "complement" of $a$, and $\subseteq$ will denote the usual partial order of "inclusion".

3. I start by recalling a well-known fact about uncountable families of sets in probability spaces.

Lemma. Let $(X, \Sigma, \nu)$ be a probability space and $\left\langle F_{\xi}\right\rangle_{\xi<\omega_{1}}$ a family of measurable sets of non-zero measure. Then there is an uncountable set $A \subseteq$ $\omega_{1}$ such that $\inf _{\xi, \eta \in A} \nu\left(E_{\xi} \cap E_{\eta}\right)>0$ (cf. [1], Theorem 6.15 ; see also [4]).

4. Notation. The core of this proof is an investigation of certain properties of the algebras $\mathfrak{B}_{\kappa}$.

(a) Much of the argument will be based on the following straightforward idea. Let $I$ be a set. If $E \subseteq\{0,1\}^{I}, J \subseteq I$ then I will say that $E$ is determined by coordinates in $J$ if $x \in E$ whenever $x \in\{0,1\}^{I}$ and there is a $y \in E$ such that $x\left\lceil J=y\left\lceil J\right.\right.$; equivalently, if there is a set $F \subseteq\{0,1\}^{J}$ such that $E=$ $\pi_{J}^{-1}[F]$, where $\pi_{J}(x)=x\left\lceil J\right.$ for $x \in\{0,1\}^{I}$; equivalently, if $E=\pi_{J}^{-1}\left[\pi_{J}[E]\right]$. Note that

(i) the family of sets determined by coordinates in $J$ is closed under complements and arbitrary intersections and unions;

(ii) if $E$ is determined by coordinates in $J$, and $J \subseteq K \subseteq I$, then $E$ is determined by coordinates in $K$;

(iii) if $E$ is determined by coordinates in $J$, and also determined by coordinates in $K$, then it is determined by coordinates in $J \cap K$.

(For if $x \in\{0,1\}^{I}, y \in E$ and $x\left\lceil J \cap K=y \mid J \cap K\right.$, define $z \in\{0,1\}^{I}$ by setting $z(i)=x(i)$ if $i \in J$, and $y(i)$ if $i \in I \backslash J$; then $z\lceil K=y\lceil K$, so $z \in E$, and $x\lceil J=z\lceil J$, so $x \in E$.) 
(b) If $E \subseteq\{0,1\}^{I}$ and $J \subseteq I$, set

$S_{J}(E)=\left\{x: x \in\{0,1\}^{I}, x+z \in E\right.$ whenever $z \in\{0,1\}^{I}$,

$$
z(i)=0 \text { for every } i \in I \backslash J\},
$$

writing + for the usual group operation on $\{0,1\}^{I}$ derived from identifying it with $\mathbb{Z}_{2}^{I}$. Observe that

(i) $S_{J}(E)$ is the largest subset of $E$ determined by coordinates in $I \backslash J$;

(ii) $S_{J} S_{K}(E)=S_{J \cup K}(E) \subseteq S_{J}(E)$;

(iii) if $K \subseteq I$ is such that $E$ is determined by coordinates in $K$ (i.e., $E=S_{I \backslash K}(E)$ ), then $S_{J}(E)$ is determined by coordinates in $K \backslash J$;

(iv) if $J$ is finite and $E$ is measurable (for the usual measure on $\{0,1\}^{I}$ ) then $S_{J}(E)$ is measurable;

(v) if $E$ is closed then $S_{J}(E)$ is closed;

(vi) if $E$ is a zero set (that is, in this context, $E$ is a closed set which is determined by coordinates in some countable set), and $J$ is countable, then $S_{J}(E)$ is a zero set.

5. Lemma. Let $I$ be a set and $\mu$ the usual measure on $X=\{0,1\}^{I}$. Let $E \subseteq X$ be a measurable set and $\left\langle I_{k}\right\rangle_{k \in \mathbb{N}}$ a disjoint sequence of subsets of $I$ all of size at most $n$. Then $\lim _{k \rightarrow \infty} \mu S_{I_{k}}(E)=\mu E$.

Proof. Let $\varepsilon>0$. There is a set $F \subseteq X$, determined by a finite set $K$ of coordinates, such that $\mu(E \triangle F) \leq \varepsilon$. Take $k_{0}$ such that $I_{k} \cap K=\emptyset$ for $k \geq k_{0}$. Then for any $k \geq k_{0}$,

$$
E \backslash S_{I_{k}}(E) \subseteq(E \backslash F) \cup \bigcup\left\{x: \exists z, z(i)=0 \forall i \in I \backslash I_{k}, x+z \in F \backslash E\right\}
$$

has measure at most $2^{n} \varepsilon$. As $\varepsilon$ is arbitrary, we have the result.

6. Without further ado, I proceed to the main result.

Theorem. Suppose that $\kappa<\mathfrak{m}$, and let $\mu$ be the usual measure on $X=\{0,1\}^{\kappa}$. Let $\left\langle\left(E_{\alpha}, E_{\alpha}^{\prime}\right)\right\rangle_{\alpha<\kappa}$ be a family of pairs of measurable subsets of $X$ such that

$$
\begin{aligned}
\left\{\alpha: \mu E_{\alpha}+\mu E_{\alpha}^{\prime} \geq 1-\varepsilon, x(\alpha)=0 \text { for every } x\right. & \in E_{\alpha}, \\
x(\alpha) & \left.=1 \text { for every } x \in E_{\alpha}^{\prime}\right\}
\end{aligned}
$$

has cardinal $\kappa$ for every $\varepsilon>0$. Then there is a set $D \subseteq \kappa$, of cardinal $\kappa$, such that

$$
\mu\left(X \cap \bigcap_{\alpha \in I} E_{\alpha} \cap \bigcap_{\beta \in J} E_{\beta}^{\prime}\right)>0
$$

for any disjoint finite sets $I, J \subseteq D$.

Proof. Part A. I first give the argument for the case in which $\kappa$ has uncountable cofinality, as this is easier, and then turn to the modifications required if $\operatorname{cf}(\kappa)=\omega$. 
(a) Write $C$ for

$\left\{\alpha: \mu E_{\alpha}+\mu E_{\alpha}^{\prime}>1 / 2, x(\alpha)=0\right.$ for every $x \in E_{\alpha}$,

$$
\left.x(\alpha)=1 \text { for every } x \in E_{\alpha}^{\prime}\right\},
$$

so that $\#(C)=\kappa$. For each $\alpha \in C$ there is a zero set $Z_{\alpha}$, determined by coordinates in $\kappa \backslash\{\alpha\}$, such that $\mu Z_{\alpha}>0$ and

$$
\left\{x: x \in Z_{\alpha}, x(\alpha)=0\right\} \subseteq E_{\alpha}, \quad\left\{x: x \in Z_{\alpha}, x(\alpha)=1\right\} \subseteq E_{\alpha}^{\prime} .
$$

(Take zero sets $F \subseteq E_{\alpha}, F^{\prime} \subseteq E_{\alpha}^{\prime}$ such that $\mu F+\mu F^{\prime}>1 / 2$. Set $Z_{\alpha}=$ $S_{\{\alpha\}}\left(F \cup F^{\prime}\right)$; this works.)

(b) (i) Let $P$ be the set of all pairs $(I, F)$ where $I \in[C]^{<\omega}, F \subseteq \bigcap_{\beta \in I} Z_{\beta}$ is a zero set determined by coordinates in $\kappa \backslash I$ and $\mu F>0$. Order $P$ by saying that $(I, F) \leq\left(I^{\prime}, F^{\prime}\right)$ if $I \subseteq I^{\prime}$ and $F^{\prime} \subseteq F$; then $P$ is a partially ordered set. Note that $q_{\alpha}=\left(\{\alpha\}, Z_{\alpha}\right)$ belongs to $P$ for every $\alpha \in C$.

(ii) $P$ is upwards-ccc. To see this, let $\left\langle\left(I_{\xi}, F_{\xi}\right)\right\rangle_{\xi<\omega_{1}}$ be a family in $P$. Then we can find an uncountable set $A \subseteq \omega_{1}$ such that

$(\alpha)\left\langle I_{\xi}\right\rangle_{\xi \in A}$ is a constant-size $\Delta$-system with root $I$ say;

( $\beta$ ) whenever $\xi<\eta$ in $A, \alpha \in I_{\xi}$ and $\beta \in I_{\eta} \backslash I$ then $\alpha<\beta$;

$(\gamma)$ whenever $\xi<\eta$ in $A$, then $F_{\xi}$ is determined by coordinates in $\kappa \backslash\left(I_{\eta} \backslash I\right)$;

$(\delta)$ there is a $\delta>0$ such that $\mu\left(F_{\xi} \cap F_{\eta}\right) \geq \delta$ for every $\xi, \eta \in A$ (using Lemma 3).

Let $\left\langle\xi_{k}\right\rangle_{k \in \mathbb{N}}$ be a strictly increasing sequence in $A$ and $\eta$ a member of $A$ greater than any $\xi_{k}$. Because \# $\left(I_{\xi_{k}} \backslash I\right)$ is the same for all $k$, Lemma 5 tells us that there is a $k$ such that

$$
\mu S_{\xi_{\xi_{k}} \backslash I}\left(F_{\eta}\right)>\mu F_{\eta}-\delta .
$$

Set $F_{\eta}^{\prime}=S_{I_{\xi_{k}} \backslash I}\left(F_{\eta}\right)$. Then

$$
\mu\left(F_{\eta} \backslash F_{\eta}^{\prime}\right)<\delta, \quad \mu\left(F_{\xi_{k}} \cap F_{\eta}^{\prime}\right)>0 .
$$

Now we know that $F_{\xi_{k}}$ is determined by coordinates in $\kappa \backslash I_{\xi_{k}}$ and also by coordinates in $\kappa \backslash\left(I_{\eta} \backslash I\right)$, and is therefore determined by coordinates in $\kappa \backslash J$, where $J=I_{\xi_{k}} \cup I_{\eta}$. Similarly, $F_{\eta}^{\prime}$ is determined by coordinates in $\left(\kappa \backslash I_{\eta}\right) \backslash\left(I_{\xi_{k}} \backslash I\right)=\kappa \backslash J$, so $F=F_{\xi_{k}} \cap F_{\eta}^{\prime}$ is also determined by coordinates in $\kappa \backslash J$, while $\mu F>0$. Finally,

$$
F \subseteq F_{\xi_{k}} \cap F_{\eta} \subseteq \bigcap_{\beta \in J} Z_{\beta} .
$$

This means that $(J, F) \in P$, and evidently it is a common upper bound for $\left(I_{\xi_{k}}, F_{\xi_{k}}\right)$ and $\left(I_{\eta}, F_{\eta}\right)$.

Thus $\left\langle\left(I_{\xi}, F_{\xi}\right)\right\rangle_{\xi<\kappa}$ is not an up-antichain. As $\left\langle\left(I_{\xi}, F_{\xi}\right)\right\rangle_{\xi<\kappa}$ is arbitrary, $P$ is upwards-ccc. 
(c) Because $\mathfrak{m}>\kappa$, there is a sequence $\left\langle R_{n}\right\rangle_{n \in \mathbb{N}}$ of upwards-directed subsets of $P$ such that $q_{\alpha} \in \bigcup_{n \in \mathbb{N}} R_{n}$ for every $\alpha \in C$ (see [3], 41Ca). Because $\#(C)=\kappa$ and $\operatorname{cf}(\kappa) \geq \omega_{1}$, there is an $n$ such that $D=\left\{\alpha: q_{\alpha} \in R_{n}\right\}$ has cardinal $\kappa$. If $I, J \in[D]^{<\omega}$ are disjoint there is a $(K, F) \in R_{n}$ which is an upper bound for $\left\{q_{\alpha}: \alpha \in I \cup J\right\}$, so that $I \cup J \subseteq K$; now $F$ is determined by coordinates in $\kappa \backslash(I \cup J)$, and

$$
\begin{array}{ll}
F \cap E_{\alpha}=F \cap Z_{\alpha} \cap E_{\alpha}=\{x: x \in F, x(\alpha)=0\} & \text { for every } \alpha \in I, \\
F \cap E_{\beta}^{\prime}=F \cap Z_{\beta} \cap E_{\beta}^{\prime}=\{x: x \in F, x(\beta)=1\} & \text { for every } \beta \in J,
\end{array}
$$

so

$$
\begin{aligned}
\mu\left(X \cap \bigcap_{\alpha \in I} E_{\alpha} \cap\right. & \left.\bigcap_{\beta \in J} E_{\beta}^{\prime}\right) \\
& \geq \mu\{x: x \in F, x(\alpha)=0 \forall \alpha \in I, x(\beta)=1 \forall \beta \in J\} \\
& =2^{-\#(I \cup J)} \mu F>0 .
\end{aligned}
$$

Thus $D$ has the declared property.

Part B. I now turn to the adaptations required if $\operatorname{cf}(\kappa) \leq \omega$. If $\kappa \leq \omega$ the result is easy and of no importance, so I leave it as an exercise for any reader who wishes to check her understanding of the hypotheses. For the case $\omega=\operatorname{cf}(\kappa)<\kappa$, start by expressing $\kappa$ as the union of a strictly increasing sequence $\left\langle\kappa_{j}\right\rangle_{j \in \mathbb{N}}$ of regular uncountable cardinals. Set

$$
\begin{aligned}
& C_{m}=\left\{\alpha: \mu E_{\alpha}+\mu E_{\alpha}^{\prime}>\frac{m+1}{m+2},\right. \\
& \left.\quad x(\alpha)=0 \text { for every } x \in E_{\alpha}, x(\alpha)=1 \text { for every } x \in E_{\alpha}^{\prime}\right\},
\end{aligned}
$$

so that $C_{m}$ has cardinal $\kappa$ for each $m \in \mathbb{N}$, by hypothesis. Let $\left\langle C_{m j}\right\rangle_{m, j \in \mathbb{N}}$ be a partition of $C=C_{0}$ such that $C_{m j} \subseteq C_{m}$ and $\#\left(C_{m j}\right)=\kappa_{j}$ for all $m, j \in \mathbb{N}$. Write $C_{j}^{\prime}=\bigcup_{m \in \mathbb{N}} C_{m j}$ for each $j$, so that $\left\langle C_{j}^{\prime}\right\rangle_{j \in \mathbb{N}}$ is disjoint and $\#\left(C_{j}^{\prime} \cap C_{m}\right)=\kappa_{j}$ for every $j, m \in \mathbb{N}$.

(a) For $j, m \in \mathbb{N}$ and $\alpha \in C_{m j}$ there is a zero set $Z_{\alpha}$, determined by coordinates in $\kappa \backslash\{\alpha\}$, such that $\mu Z_{\alpha}>m /(m+2)$ and $\left\{x: x \in Z_{\alpha}, x(\alpha)=\right.$ $0\} \subseteq E_{\alpha},\left\{x: x \in Z_{\alpha}, x(\alpha)=1\right\} \subseteq E_{\alpha}^{\prime}$. (In (A-a) above, take $F, F^{\prime}$ such that $\mu F+\mu F^{\prime}>(m+1) /(m+2)$ and continue as before.)

(b) Define the partially ordered set $P$ as in (A-b-i); as in (A-b-ii), $P$ is ccc. Once again, set $q_{\alpha}=\left(\{\alpha\}, Z_{\alpha}\right)$ for $\alpha \in C$.

(c) Now for the new idea.

(i) For any $m, j \in \mathbb{N}$ there is a finite set $I_{m j}^{*} \subseteq \kappa$ such that whenever $(I, F) \in P$ and $I \cap I_{m j}^{*}=\emptyset, \#(I) \leq m, \mu F \geq 2 /(m+2)$ then

$$
A_{m j}(I, F)=\left\{\alpha: \alpha \in C_{m j},(I, F) \text { and } q_{\alpha} \text { are compatible in } P\right\}
$$


has cardinal $\kappa_{j}$. For suppose, if possible, otherwise. Then we can find a sequence $\left\langle\left(I_{k}, F_{k}\right)\right\rangle_{k \in \mathbb{N}}$ in $P$ such that $\#\left(I_{k}\right) \leq m, \mu F_{k} \geq 2 /(m+2)$, $\#\left(A_{m j}\left(I_{k}, F_{k}\right)\right)<\kappa_{j}$ for every $k$ and $\left\langle I_{k}\right\rangle_{k \in \mathbb{N}}$ is disjoint. For each $k$, let $J_{k} \subseteq$ $\kappa$ be a countable set such that $F_{k}$ is determined by coordinates in $J_{k}$. Because $\kappa_{j}$ has uncountable cofinality, there is an $\alpha \in C_{m j} \backslash \bigcup_{k \in \mathbb{N}}\left(A_{m j}\left(I_{k}, F_{k}\right) \cup J_{k}\right)$. But now Lemma 5 tells us that

$$
\lim _{k \rightarrow \infty} \mu S_{I_{k}}\left(Z_{\alpha}\right)=\mu Z_{\alpha}>\frac{m}{m+2},
$$

so there is some $k$ such that $\mu S_{I_{k}}\left(Z_{\alpha}\right)>m /(m+2)$. Set $F^{\prime}=F_{k} \cap S_{I_{k}}\left(Z_{\alpha}\right)$. Then

$$
\mu F^{\prime} \geq \mu F_{k}+\mu S_{I_{k}}\left(Z_{\alpha}\right)-1>0,
$$

and $F^{\prime}$ is determined by coordinates in $\kappa \backslash\left(I_{k} \cup\{\alpha\}\right)$, so $\left(I_{k} \cup\{\alpha\}, F^{\prime}\right)$ witnesses that $\left(I_{k}, F_{k}\right)$ and $q_{\alpha}$ are compatible, which is supposed to be impossible.

(ii) Set $I^{*}=\bigcup_{m, j \in \mathbb{N}} I_{m j}^{*}$, so that $I^{*}$ is countable. Set $P^{*}=\{(I, F)$ : $\left.(I, F) \in P, I \cap I^{*}=\emptyset\right\}$. Then two members of $P^{*}$ are compatible in $P^{*}$ iff they are compatible in $P$ (if $\left(I_{1}, F_{1}\right),\left(I_{2}, F_{2}\right)$ belong to $P^{*}$ and have a common upper bound $(I, F) \in P$, then $\left(I_{1} \cup I_{2}, F\right) \in P^{*}$ is still an upper bound), so $P^{*}$ is also ccc.

(iii) Enumerate each $C_{j}^{\prime}$ as $\left\langle\gamma_{\xi}\right\rangle_{\xi<\kappa_{j}}$. For $\beta<\kappa_{j}$, set

$$
Q_{j \beta}=\left\{(I, F):(I, F) \in P^{*}, \exists \xi, \beta \leq \xi<\kappa_{j}, \gamma_{\xi} \in I\right\} .
$$

Then $Q_{j \beta}$ is cofinal with $P^{*}$. For if $(I, F) \in P^{*}$, there is some $m \in \mathbb{N}$ such that $\#(I) \leq m$ and $\mu F \geq 2 /(m+2)$. Because $I \cap I_{m j}^{*}=\emptyset, A_{m j}(I, F) \subseteq C_{j}^{\prime}$ has cardinal $\kappa_{j}$ and there must be some $\xi \geq \beta$ such that $\gamma_{\xi} \in A_{m j}(I, F) \backslash I^{*}$. But now $(I, F), q_{\gamma_{\xi}}$ have a common upper bound in $P$ and therefore a common upper bound in $P^{*}$, which is a member of $Q_{j \beta}$ greater than or equal to $(I, F)$.

(iv) Because $\kappa<\mathfrak{m}$, there is an upwards-directed set $R \subseteq P^{*}$ meeting every $Q_{j \beta}$. Set $D=\bigcup\{I:(I, F) \in R\}$. Because $R$ meets every $Q_{j \beta}$, we have $\#\left(D \cap C_{j}^{\prime}\right)=\kappa_{j}$ for every $j$ and $\#(D)=\kappa$. And $D$ has the property required by the theorem, just as in (A-c) above.

7. Corollary. Suppose that $\omega \leq \kappa<\mathfrak{m}$. Let $(\mathfrak{A}, \bar{\mu})$ be a probability algebra. Suppose that $\left\langle a_{\alpha}\right\rangle_{\alpha<\kappa}$ is a stochastically independent family of elements of measure $1 / 2$, and that for each $\alpha<\kappa$ we are given elements $e_{\alpha} \subseteq a_{\alpha}$, $e_{\alpha}^{\prime} \subseteq 1 \backslash a_{\alpha}$ such that $\left\{\alpha: \bar{\mu} e_{\alpha}+\bar{\mu} e_{\alpha}^{\prime} \geq 1-\varepsilon\right\}$ has cardinal $\kappa$ for every $\varepsilon>0$. Then there is a set $D \subseteq \kappa$, of cardinal $\kappa$, such that $\inf _{\alpha \in I} e_{\alpha} \cap \inf _{\beta \in J} e_{\beta}^{\prime} \neq 0$ for all disjoint finite $I, J \subseteq D$.

Pr o of. Let $\mathfrak{A}_{0}$ be the closed subalgebra of $\mathfrak{A}$ generated by $\left\{a_{\alpha}: \alpha<\kappa\right\}$, so that $\mathfrak{A}_{0}$ is isomorphic (as measure algebra) to $\mathfrak{B}_{\kappa}$. Let $C \subseteq \kappa$ be a set such that $C$ and $\kappa \backslash C$ both have cardinal $\kappa$; let $h: \kappa \rightarrow C$ be a bijection. 
Then we have a measure-preserving Boolean homomorphism $\pi_{0}: \mathfrak{A}_{0} \rightarrow \mathfrak{B}_{\kappa}$ defined by saying that $\pi a_{\alpha}=b_{h(\alpha)}$, where $b_{\beta} \in \mathfrak{B}_{\kappa}$ is the equivalence class of $G_{\beta}=\{x: x(\beta)=0\}$. We can identify $\mathfrak{B}_{\kappa}$ with the probability algebra free product $\pi_{0}[\mathfrak{A}] \widehat{\oplus} \mathfrak{C}$, where $\mathfrak{C}$ is the closed subalgebra generated by $\left\{b_{\beta}: \beta \in \kappa \backslash C\right\}$ (cf. [5], 2.25b). Let $\mathfrak{A}_{1}$ be the closed subalgebra of $\mathfrak{A}$ generated by $\left\{a_{\alpha}: \alpha<\kappa\right\} \cup\left\{e_{\alpha}: \alpha<\kappa\right\} \cup\left\{e_{\alpha}^{\prime}: \alpha<\kappa\right\}$. Then $\pi_{0}$ has an extension to a measure-preserving Boolean homomorphism $\pi_{1}: \mathfrak{A}_{1} \rightarrow \mathfrak{B}_{\kappa}$ (see [5], 3.11a).

Let $\mu$ be the usual measure on $\{0,1\}^{\kappa}$. For $\beta \in C$, take $E_{\beta} \subseteq G_{\beta}$, $E_{\beta}^{\prime} \subseteq\{0,1\}^{\kappa} \backslash G_{\beta}$ such that $E_{\beta}^{\bullet}=\pi_{1} e_{\alpha},\left(E_{\beta}^{\prime}\right)^{\bullet}=\pi_{1} e_{\alpha}^{\prime}$, where $\alpha=h^{-1}(\beta)$. For $\beta \in \kappa \backslash C$, set $E_{\beta}=E_{\beta}^{\prime}=\emptyset$. Then

$$
\#\left(\left\{\beta: \mu E_{\beta}+\mu E_{\beta}^{\prime} \geq 1-\varepsilon\right\}\right)=\#\left(\left\{\alpha: \bar{\mu} e_{\alpha}+\bar{\mu} e_{\alpha}^{\prime} \geq 1-\varepsilon\right\}\right)=\kappa
$$

for every $\varepsilon>0$.

Applying Theorem 6, there is a set $D_{0} \in[\kappa]^{\kappa}$ such that $\mu\left(\bigcap_{\alpha \in I} E_{\alpha} \cap\right.$ $\left.\bigcap_{\beta \in J} E_{\beta}^{\prime}\right)>0$ for all disjoint finite $I, J \subseteq D$, that is, $\bar{\mu}\left(\inf _{\alpha \in I} e_{\alpha} \cap \inf _{\beta \in J} e_{\beta}^{\prime}\right)$ $>0$ for all disjoint finite $I, J \subseteq h^{-1}\left[D_{0}\right]$. Of course, $D_{0} \subseteq C$. So we take $D=h^{-1}\left[D_{0}\right]$.

8. Corollary. Suppose that $\omega \leq \kappa<\mathfrak{m}$. Let $(X, \Sigma, \mu)$ be a probability space. Suppose that $\left\langle G_{\alpha}\right\rangle_{\alpha<\kappa}$ is a stochastically independent family of elements of measure $1 / 2$, and that for each $\alpha<\kappa$ we have measurable sets $E_{\alpha} \subseteq G_{\alpha}, E_{\alpha}^{\prime} \subseteq X \backslash G_{\alpha}$ such that $\left\{\alpha: \mu E_{\alpha}+\mu E_{\alpha}^{\prime} \geq 1-\varepsilon\right\}$ has cardinal $\kappa$ for every $\varepsilon>0$. Then there is a set $D \subseteq \kappa$, of cardinal $\kappa$, such that $\mu\left(X \cap \bigcap_{\alpha \in I} E_{\alpha} \cap \bigcap_{\beta \in J} E_{\beta}^{\prime}\right)>0$ for all disjoint finite $I, J \subseteq D$.

Proof. Apply Corollary 7 with $(\mathfrak{A}, \bar{\mu})$ the measure algebra of $\mu, a_{\alpha}=$ $G_{\alpha}^{\bullet}, e_{\alpha}=E_{\alpha}^{\bullet}$ and $e_{\alpha}^{\prime}=\left(E_{\alpha}^{\prime}\right)^{\bullet}$ for each $\alpha$.

9. Theorem. Suppose that $\omega \leq \kappa<\mathfrak{m}$. Let $(X, \mathfrak{T})$ be a compact Hausdorff space. Then the following are equivalent:

(i) there is a Radon probability measure $\mu$ on $X$ with measure algebra isomorphic to $\mathfrak{B}_{\kappa}$;

(ii) there is a continuous surjection from $X$ onto $[0,1]^{\kappa}$.

Proof. The implication (ii) $\Rightarrow(\mathrm{i})$ is discussed in $\S 1$ above (and does not depend on the assumption $\mathfrak{m}>\kappa)$; this proof will therefore address (i) $\Rightarrow$ (ii). Let $\left\langle G_{\alpha}\right\rangle_{\alpha<\kappa}$ be a stochastically independent family of sets of measure $1 / 2$. Let $\left\langle C_{m}\right\rangle_{m \in \mathbb{N}}$ be a partition of $\kappa$ into sets of size $\kappa$. For each $\alpha$, we can find compact sets $E_{\alpha} \subseteq G_{\alpha}, E_{\alpha}^{\prime} \subseteq X \backslash G_{\alpha}$ such that $\mu E_{\alpha}+\mu E_{\alpha}^{\prime}>m /(m+1)$ if $\alpha \in C_{m}$. By Corollary 8, there is a set $D \in[\kappa]^{\kappa}$ such that $\mu\left(X \cap \bigcap_{\alpha \in I} E_{\alpha} \cap\right.$ $\left.\bigcap_{\beta \in J} E_{\beta}^{\prime}\right)>0$ for all disjoint finite $I, J \subseteq D$. In particular, $\bigcap_{\alpha \in I} E_{\alpha} \cap$ $\bigcap_{\beta \in J} E_{\beta}^{\prime}$ is non-empty for all disjoint finite $I, J \subseteq D$; that is, $\left\langle\left(E_{\alpha}, E_{\alpha}^{\prime}\right)\right\rangle_{\alpha \in D}$ 
is independent in the sense of [7]. By Lemma 2.1 of $[7],[0,1]^{\kappa}$ is a continuous image of $X$.

10. Remarks. (a) If you look back at the demonstration that the partially ordered set $P$ is ccc, in (A-b-ii) of the proof of Theorem 6, you will see that what I show there is that, given a family $\left\langle p_{\xi}\right\rangle_{\xi<\omega_{1}}$ in $P$, there is an uncountable set $A \subseteq \omega_{1}$ such that whenever $\left\langle\xi_{k}\right\rangle_{k \in \mathbb{N}}$ is a strictly increasing sequence in $A$, and $\eta \in A$ is greater than any $\xi_{k}$, then there is some $k$ such that $p_{\xi_{k}}, p_{\eta}$ are compatible in $P$. But this means that there must be an uncountable $A^{\prime} \subseteq A$ such that $p_{\xi}, p_{\eta}$ are compatible for all $\xi, \eta \in A^{\prime}$, by the Erdős-Dushnik-Miller theorem $([3]$, A2K). Accordingly, $P$ satisfies Knaster's condition $([3], 11 \mathrm{~A})$. In all the results of this paper, therefore, we may replace " $\mathfrak{m}>\kappa$ " by " $\mathfrak{m}_{\mathrm{K}}>\kappa$ ", where $\mathfrak{m}_{\mathrm{K}} \geq \mathfrak{m}$ is the cardinal associated with "Martin's axiom for partially ordered sets with Knaster's condition" $([3], 11 \mathrm{D})$. (In part (A-c) of the proof of Theorem 6 , we must now use 31B of [3] in place of $41 \mathrm{C}$.)

(b) I note that when $\operatorname{cf}(\kappa) \geq \omega_{1}$ then the hypotheses of $\S \S 6-8$ are unnecessarily elaborate. Part A of the proof of Theorem 6 demands only that $\mu E_{\alpha}+\mu E_{\alpha}^{\prime}>1 / 2$ for $\kappa$ indices $\alpha$. So in Corollary 7, for instance, we have:

Suppose that $\kappa<\mathfrak{m}_{\mathrm{K}}$ is a cardinal of uncountable cofinality. Let $(\mathfrak{A}, \bar{\mu})$ be a probability algebra. Suppose that $\left\langle a_{\alpha}\right\rangle_{\alpha<\kappa}$ is a stochastically independent family of elements of measure $1 / 2$ in $\mathfrak{A}$, and that for each $\alpha<\kappa$ we are given elements $e_{\alpha} \subseteq a_{\alpha}, e_{\alpha}^{\prime} \subseteq 1 \backslash a_{\alpha}$ such that $\bar{\mu} e_{\alpha}+\bar{\mu} e_{\alpha}^{\prime}>1 / 2$ for every $\alpha$. Then there is a set $D \subseteq \kappa$, of cardinal $\kappa$, such that $\inf _{\alpha \in I} e_{\alpha} \cap \inf _{\beta \in J} e_{\beta}^{\prime} \neq 0$ for all disjoint finite $I, J \subseteq D$.

Acknowledgements. I should like to mention here two of the essential elements leading to the discoveries described above: first, a preview of [14], sent to me by its author; second, the courtesy and stamina of M. R. Burke, M. Džamonja and M. Foreman in the face of the original exposition. As a corollary, I must thank the Mathematics Department of the University of Wisconsin for making it possible for us to gather there.

\section{References}

[1] W. W. Comfort and S. Negrepontis, Chain Conditions in Topology, Cambridge Univ. Press, 1982.

[2] M. Džamonja and K. Kunen, Measures on compact HS spaces, Fund. Math. 143 (1993), 41-54.

[3] D. H. Fremlin, Consequences of Martin's Axiom, Cambridge Univ. Press, 1984.

[4] - Large correlated families of positive random variables, Math. Proc. Cambridge Philos. Soc. 103 (1988), 147-162.

[5] -, Measure algebras, pp. 877-980 in [13]. 
[6] D. H. Fremlin, Real-valued-measurable cardinals, pp. 151-304 in [9].

[7] R. G. Haydon, On Banach spaces which contain $\ell^{1}(\tau)$ and types of measures on compact spaces, Israel J. Math. 28 (1977), 313-324.

[8] -, On dual $L^{1}$-spaces and injective bidual Banach spaces, Israel J. Math. 31 (1978), $142-152$.

[9] H. Judah (ed.), Set Theory of the Reals, Israel Math. Conf. Proc. 6, Bar-Ilan Univ., 1993.

[10] K. Kunen, A compact L-space under CH, Topology Appl. 12 (1981), 283-287.

[11] K. Kunen and J. van Mill, Measures on Corson compact spaces, Fund. Math. 147 (1995), 61-72.

[12] D. Maharam, On homogeneous measure algebras, Proc. Nat. Acad. Sci. U.S.A. 28 (1942), 108-111.

[13] J. D. Monk (ed.), Handbook of Boolean Algebras, North-Holland, 1989.

[14] G. Plebanek, Nonseparable Radon measures and small compact spaces, Fund. Math. 153 (1997), 25-40.

Department of Mathematics

University of Essex

Colchester, England

E-mail: fremdh@essex.ac.uk 